\title{
Inhibition of the NMDA receptor protects the rat sciatic nerve against ischemia/reperfusion injury
}

\author{
TIE KE $\mathrm{KE}^{1-3 *},{\mathrm{RENBIN} \mathrm{LI}^{4 *} \text { and WENCHANG CHEN }}^{1}$ \\ ${ }^{1}$ Department of Emergency Surgery, Fujian Provincial Hospital; ${ }^{2}$ Emergency Center of Fujian Province; \\ ${ }^{3}$ Provincial Clinical Medical College, Fujian Medical University, Fuzhou, Fujian 350001; \\ ${ }^{4}$ Department of Orthopedics, The Affiliated Fuzhou Second Hospital, Xiamen University, Fuzhou, Fujian 350007, P.R. China
}

Received November 17, 2014; Accepted November 25, 2015

DOI: $10.3892 /$ etm.2016.3148

\begin{abstract}
Inhibition of the N-methyl-D-aspartate (NMDA) receptor by $\mathrm{MK}-801$ reduces ischemia/reperfusion (I/R) injury in the central nervous system. However, few previous studies have evaluated the neuroprotective effects of MK-801 against peripheral I/R injury. The present study aimed to investigate the protective effects of MK-801 pretreatment against I/R injury in the rat sciatic nerve (SN). Sprague-Dawley rats were subjected to a sham surgery $(n=8)$ or to a 5 -h ischemic insult by femoral artery clamping (I/R and I/R+MK-801 groups; $\mathrm{n}=48$ per group). I/R+MK-801 rats were intraperitoneally injected with MK-801 $(0.5 \mathrm{ml}$ or $1 \mathrm{mg} / \mathrm{kg})$ at $15 \mathrm{~min}$ prior to reperfusion. The rats were sacrificed at $0,6,12,24,72 \mathrm{~h}$, or 7 days following reperfusion. Plasma malondialdehyde (MDA) and nitric oxide (NO) concentrations, and $\mathrm{SN}$ inducible NO synthase (iNOS) protein expression levels, were measured using colorimetry. In addition, the protein expression levels of tumor necrosis factor- $\alpha$ (TNF- $\alpha$ ) were measured using immunohistochemistry, and histological analyses of the rat SN were conducted using light and electron microscopy. Alterations in the mRNA expression levels of TNF- $\alpha$ and TNF- $\alpha$ converting enzyme (TACE) in the rat $\mathrm{SN}$ were detected using reverse transcription-quantitative polymerase chain reaction. In the I/R group, plasma concentrations of NO $(175.3 \pm 4.2 \mu \mathrm{mol} / \mathrm{l})$ and MDA $(16.2 \pm 1.9 \mathrm{mmol} / \mathrm{l})$, and the levels of iNOS $(2.5 \pm 0.3)$ in the $\mathrm{SN}$, peaked at $24 \mathrm{~h}$ post-reperfusion. At $24 \mathrm{~h}$, pretreatment with MK-801 significantly reduced plasma NO $(107.3 \pm 3.6 \mu \mathrm{mol} / \mathrm{l})$ and MDA $(11.8 \pm 1.6 \mathrm{mmol} / \mathrm{l})$, and SN iNOS $(1.65 \pm 0.2)$ levels (all $\mathrm{P}<0.01)$. The mRNA expression levels of
\end{abstract}

Correspondence to: Dr Tie Ke, Department of Emergency Surgery, Fujian Provincial Hospital, Fujian Medical University, 134 East Street, Fuzhou, Fujian 350001, P.R. China

E-mail: medsciketie@126.com

*Contributed equally

Key words: sciatic nerve, ischemia/reperfusion, tumor necrosis factor- $\alpha$ converting enzyme, N-methyl-D-aspartate receptor antagonist, inducible nitric oxide synthase, tumor necrosis factor- $\alpha$
TNF- $\alpha$ and TACE in the SN were significantly reduced in the I/R+MK-801 group, as compared with the I/R group $(\mathrm{P}<0.05)$. Furthermore, MK-801 pretreatment was shown to have alleviated histological signs of I/R injury, including immune cell infiltration and axon demyelination. The results of the present study suggested that pretreatment with MK-801 may alleviate I/R injury of the SN by inhibiting the activation of TNF- $\alpha$ and reducing the levels of iNOS in the SN.

\section{Introduction}

Peripheral nerve injury is a complication that is commonly observed following orthopedic surgery or trauma (1-3). Peripheral nerve ischemia and reperfusion (I/R), which may be the cause of nerve injury, may occur following incision for pressure-relief in extremity intracompartment syndrome, during compression for repair of vascular rupture or from nerve trunk oppression (4). Numerous interacting mechanisms may contribute to axonal degeneration following I/R, including immune cell activation/infiltration, calcium dysregulation, and free radical generation (5).

In the central nervous system (CNS), glutamate release and the concomitant overstimulation of synaptic glutamate receptors, particularly the N-methyl-D-aspartate (NMDA) subtype, is a critical early event in I/R injury (6). The involvement of glutamate receptors in I/R injury has been extensively studied in the CNS (5); however, peripheral nerves also express glutamate receptors, which may contribute to axonal injury and nerve dysfunction, including initiation of neuropathic pain (7-13). Oxidative stress is a hallmark of peripheral nervous system (PNS) axon I/R injury, and occurs due to the generation of free-radicals $(4,14-17)$. Free-radical scavengers have previously been shown to mitigate I/R injury in the PNS (18). Activation of nitric oxide (NO) synthase (NOS) may induce oxidative damage during I/R (19-22); NO is highly reactive and may form toxic reactive nitrogen species during reperfusion $(23,24)$. Conversely, as a major vascular relaxation factor, NO may also protect against ischemic injury (25).

The pro-inflammatory cytokine tumor necrosis factor (TNF)- $\alpha$ has previously been shown to be upregulated in ischemic peripheral nerves $(26,27)$. Conversely, downregulation of TNF- $\alpha$ expression levels appeared to protect peripheral nerve integrity and mitigate neurological sequela (28). TNF- $\alpha$ activity 
is influenced by the TNF- $\alpha$ converting enzyme (TACE) (29). Therefore, TACE has been considered a potential therapeutic target for CNS inflammatory disorders (29); however, there is limited information regarding its function in the PNS. In a previous study, stress-induced increases in TACE activity, and increased expression levels of TNF- $\alpha$, were blocked by the noncompetitive NMDA receptor antagonist MK-801 (30). This was consistent with the results of previous studies, which demonstrated neuroprotective effects for MK-801 in various models of strokes $(31,32)$.

The present study hypothesized that MK-801 may protect peripheral nerves against I/R injury by inhibiting the activation of TNF- $\alpha$ by TACE, and thereby suppressing inflammation-mediated damage, and by inhibiting the activation of iNOS, and thereby suppressing oxidative damage. Therefore, the present study aimed to investigate the effects of MK-801 on the expression levels of TNF- $\alpha$, TACE and iNOS, and the peripheral nerve histology, in a rat model of I/R injury.

\section{Materials and methods}

Animals. A total of 104 male Sprague-Dawley rats (age, 8-9 weeks; weight, 250-300 g) were supplied by the Animal Laboratory of the Fujian Medical University (Fuzhou, China). The rats were maintained under a 12-h light/dark cycle at $20-25^{\circ} \mathrm{C}$ and $50 \pm 5 \%$ humidity, with ad libitum access to food and water. The rats were acclimatized for 1 week prior to the experiments. All procedures and animal experiments were approved by the Animal Care and Use Committee of the Fujian Medical University, and were conducted in accordance with all state regulations.

Rat model of sciatic nerve I/R injury. The rat model of SN I/R injury was established as described in a previous study (33). Briefly, the rats were fasted for $12 \mathrm{~h}$, with ad libitum access to water, prior to experiments. Subsequently, the rats were anesthetized by intraperitoneal injection with $3 \%$ pentobarbital sodium (30 mg/kg; Maixin Biological Technology Development Co., Fuzhou, China) and placed onto the operating board in the supine position. The groin on the right side was depilated using a knife and disinfected with iodine complex, after which a bevel cut was made in the right lower quadrant. In order to induce lower limb ischemia, the right arteria iliaca communis, femoral artery and arteria circumflexa iliaca superficialis were clamped for $5 \mathrm{~h}$ with micro-artery forceps using an extraperitoneal approach.

Animal grouping. The rats were randomly divided into the following groups: i) Sham group $(n=8)$; ii) I/R group $(n=48)$; and iii) I/R+MK-801 group $(n=48)$. The rats in the sham group were subjected to anesthesia, skin preparation involving depilation and disinfection with iodine, and bevel cutting into the right groin; however, the arteries were not clamped. Blood samples $(8 \mathrm{ml})$ were obtained from all rats and the right $\mathrm{SN}$ were collected $24 \mathrm{~h}$ after stitching of the wound.

The rats in the I/R group underwent I/R and were injected intraperitoneally with $0.5 \mathrm{ml}$ normal saline at $15 \mathrm{~min}$ prior to reperfusion. The rats in the I/R+MK-801 group underwent $\mathrm{I} / \mathrm{R}$ and were intraperitoneally injected with $0.5 \mathrm{ml} \mathrm{MK}-801$ (1 mg/kg; Sigma-Aldrich, St. Louis, MO, USA) at 15 min prior to reperfusion. The rats in the I/R and I/R+MK-801 groups were divided into six subgroups ( $\mathrm{n}=8$ per subgroup), depending on the time at which they were sacrificed following reperfusion $(0,6,12,24,72 \mathrm{~h}$, or 7 days). Rats in the same subgroup did not vary in body weight by $>10 \mathrm{~g}$. Blood samples were collected from the jugular vein immediately prior to sacrifice. The rats were sacrificed by acute blood loss following anesthetization with $10 \mathrm{mg} / \mathrm{kg}$ xylazine hydrochloride (Maixin Biological Technology Development Co.) and $70 \mathrm{mg} / \mathrm{kg}$ ketamine (Maixin Biological Technology Development Co.). Following sacrifice, a 5 -cm section of the ipsilateral $\mathrm{SN}$, ending $2 \mathrm{~cm}$ above the right knee joint, was removed.

Detection of iNOS activity in SN tissues. A 0.5-g sample of $\mathrm{SN}$ was rinsed with ice-cold normal saline for removal of blood, dried with filter paper, and homogenized in ice-cold normal saline (dilution, $1: 9)$ containing $0.86 \%$ sodium chloride. The concentration of iNOS was detected using a Nitric Oxide Synthase typed assay kit (A014-1; Nanjing Jiancheng Bioengineering Institute, Nanjing, China) and a type 721 spectrophotometer (Shanghai Precision \& Scientific Instrument Co., Ltd., Shanghai, China). The protein concentration was quantified using a Bicinchoninic Acid Protein Assay kit (Beyotime Institute of Biotechnology, Nantong, China), according to the manufacturer's instructions. Activity of iNOS is expressed per $\mathrm{mg}$ of proteins.

Plasma NO levels. The plasma concentration of NO was determined using a commercial NO Assay kit (A012; Nanjing Jiancheng Bioengineering Institute), according to the manufacturer's protocol. Absorbance was measured at $550 \mathrm{~nm}$ using the type 721 spectrophotometer (Shanghai Precise Scientific Instrument Co., Ltd.).

Plasma malondialdehyde (MDA) levels. The plasma concentration of MDA was detected in $0.1 \mathrm{ml}$ plasma samples using the MDA Assay kit (A003-1; Nanjing Jiancheng Bioengineering Institute), according to the manufacturer's protocol. Absorbance was measured at $721 \mathrm{~nm}$ using a type 721 spectrophotometer (Shanghai Precise Scientific Instrument Co., Ltd.).

Hematoxylin \& eosin $(H \& E)$ staining. SNs were collected, fixed with $10 \%$ formaldehyde and embedded in paraffin. Slices $(4 \mu \mathrm{m})$ were prepared and heated at $60^{\circ} \mathrm{C}$ for $6 \mathrm{~h}$ to dry. Following removal of paraffin, the slices were stained with H\&E (Thermo Fisher Scientific, Inc., Waltham, MA, USA) and visualized under an inverted phase contrast microscope (Olympus Corporation, Tokyo, Japan).

Immunohistochemical analysis. Protein expression levels of TNF- $\alpha$ in the SN were measured using the Elivision TM Plus Polymer HRP (Mouse/Rabbit) Immunohistochemistry kit (Maixin Biological Technology Development Co.) based on streptavidin-biotin-peroxidase visualization (Wuhan Boster Bio-Engineering Co., Ltd., Wuhan, China). Tissue slices were incubated with rabbit anti-mouse TNF- $\alpha$ polyclonal antibody (1:100; BA14901; Wuhan Boster Bio-Engineering Co., Ltd.) at $4^{\circ} \mathrm{C}$ overnight. After washing in phosphate-buffered saline (Maixin Biological Technology Development Co.), labeled slices were incubated with biotin-conjugated goat anti-mouse 
Table I. Effects of MK-801 on siatic nerve iNOS activity, and plasma NO and MDA levels following I/R injury.

Time post-reperfusion

\begin{tabular}{lcccccc}
\cline { 2 - 6 } Marker & $0 \mathrm{~h}$ & $6 \mathrm{~h}$ & $12 \mathrm{~h}$ & $24 \mathrm{~h}$ & $72 \mathrm{~h}$ & 7 days \\
\hline iNOS (U/mg) & & & & & \\
Sham & $0.23 \pm 0.11$ & $0.25 \pm 0.08$ & $0.30 \pm 0.15$ & $0.27 \pm 0.10$ & $0.23 \pm 0.07$ & $0.19 \pm 0.05$ \\
I/R & $0.29 \pm 0.10$ & $0.62 \pm 0.13^{\mathrm{a}}$ & $1.68 \pm 0.18^{\mathrm{b}}$ & $2.45 \pm 0.25^{\mathrm{b}}$ & $1.80 \pm 0.21^{\mathrm{b}}$ & $0.68 \pm 0.25^{\mathrm{b}}$ \\
I/R+MK-801 & $0.26 \pm 0.13$ & $0.47 \pm 0.15^{\mathrm{a}}$ & $1.25 \pm 0.10^{\mathrm{b}, \mathrm{c}}$ & $1.65 \pm 0.23^{\mathrm{b}, \mathrm{d}}$ & $1.36 \pm 0.19^{\mathrm{b}, \mathrm{c}}$ & $0.49 \pm 0.15^{\mathrm{a}, \mathrm{c}}$ \\
NO $(\mu \mathrm{mol} / \mathrm{l})$ & & & & & \\
Sham & $38.35 \pm 5.12$ & $43.28 \pm 6.17$ & $50.63 \pm 8.25$ & $48.43 \pm 9.52$ & $42.83 \pm 5.92$ & $35.74 \pm 6.29$ \\
I/R & $53.64 \pm 3.51^{\mathrm{a}}$ & $75.16 \pm 2.65^{\mathrm{b}}$ & $127.85 \pm 3.18^{\mathrm{b}}$ & $175.29 \pm 4.15^{\mathrm{b}}$ & $97.74 \pm 2.18^{\mathrm{b}}$ & $75.91 \pm 1.76^{\mathrm{b}}$ \\
I/R+MK-801 & $48.03 \pm 4.13^{\mathrm{a}}$ & $60.15 \pm 1.77^{\mathrm{b}, \mathrm{c}}$ & $84.78 \pm 3.78^{\mathrm{b}, \mathrm{d}}$ & $107.25 \pm 3.62^{\mathrm{b}, \mathrm{d}}$ & $63.65 \pm 1.47^{\mathrm{b}, \mathrm{c}}$ & $55.28 \pm 2.76^{\mathrm{a}, \mathrm{c}}$ \\
MDA $(\mu \mathrm{mol} / \mathrm{l})$ & & & & & \\
Sham & $4.52 \pm 0.62$ & $4.81 \pm 1.18$ & $6.12 \pm 1.30$ & $5.34 \pm 1.11$ & $4.82 \pm 0.96$ & $4.23 \pm 0.45$ \\
I/R & $6.30 \pm 0.73$ & $9.73 \pm 1.21^{\mathrm{a}}$ & $12.54 \pm 1.46^{\mathrm{b}}$ & $16.23 \pm 1.92^{\mathrm{b}}$ & $10.63 \pm 1.23^{\mathrm{b}}$ & $6.56 \pm 0.5^{\mathrm{a}}$ \\
I/R+MK-801 & $5.95 \pm 0.65$ & $7.22 \pm 1.05^{\mathrm{a}, \mathrm{c}}$ & $9.08 \pm 1.20^{\mathrm{b}, \mathrm{c}}$ & $11.83 \pm 1.64^{\mathrm{b}, \mathrm{d}}$ & $7.95 \pm 0.81^{\mathrm{a}, \mathrm{c}}$ & $5.14 \pm 0.52$
\end{tabular}

Data are presented as the mean \pm standard deviation $(\mathrm{n}=6) .{ }^{\mathrm{a}} \mathrm{P}<0.05,{ }^{\mathrm{b}} \mathrm{P}<0.01 \mathrm{vs}$. the sham-operated group; ${ }^{\mathrm{C}} \mathrm{P}<0.05$, ${ }^{\mathrm{d}} \mathrm{P}<0.01 \mathrm{vs}$. the I/R group. $\mathrm{I} / \mathrm{R}$, ischemia/reperfusion; iNOS, inducible-nitric oxide (NO) synthase; MDA, malondialdehyde.

immunoglobulin G polyclonal antibody (1:10,000; BA1001; Wuhan Boster Bio-Engineering Co., Ltd.) at $25^{\circ} \mathrm{C}$ for $20 \mathrm{~min}$, followed by incubation with a streptavidin-biotin-peroxidase complex for $20 \mathrm{~min}$ at $25^{\circ} \mathrm{C}$. Staining was visualized by incubating the tissue sections with $99 \%$ diaminobenzidine solution (Maixin Biological Technology Development Co.) at room temperature for 3-5 min, followed by counterstaining with hematoxylin and mounting with fluorescent mounting medium (DakoCytomation, Glostrup, Denmark). Immunostaining was semi-quantified using the Image-Pro Plus software, version 5 (Media Cybernetics, Inc., Rockville, MD, USA). Total cell numbers and the fluorescence intensity of each cell were counted and quantified in six separate fields (75-100 cells/field) for each of the conditions. The relative fluorescence intensity was calculated by dividing the total integrated optical density (IOD) by the total number of cells in each field. Mean fluorescence intensity measurements were. All sections were examined by a pathologist blinded to the grouping.

Electron microscopy. SNs were fixed in a solution containing 25\% glutaric dialdehyde (Maixin Biological Technology Development Co.) and $1.5 \%$ paraformaldehyde (Nanjing Sen Beijia biotechnology Co., Ltd., Nanjing, China). Following fixation with $1 \%$ osmium tetroxide (Maixin Biological Technology Development Co.), and dehydration with ethanol and acetone, the SNs were embedded in epoxy resin 618 (Maixin Biological Technology Development Co.). Ultrathin sections were prepared with a LKB-5 Ultramicrotome (GE Healthcare Life Sciences, Uppsala, Sweden), double-stained with uranyl acetate and calcium citrate (both Maixin Biological Technology Development Co.), and examined using an HU-12A Transmission Electron Microscope (Hitachi, Ltd., Tokyo, Japan).

Semi-quantitative reverse transcription-polymerase chain reaction (RT-PCR). Total RNA was isolated from $\mathrm{SN}$ tissues
(0.1 mg) using TRIzol ${ }^{\circledR}$ reagent (Invitrogen; Thermo Fisher Scientific, Inc.). RNA purity was determined by measuring the absorbance ratio at 260 and $280 \mathrm{~nm}$ (A260/A280) using a NanoVue UV spectrophotometer (GE Healthcare Life Sciences). cDNA was synthesized using the PrimeScript First Strand cDNA Synthesis kit (Takara Biotechnology Co., Ltd., Dalian, China) according to the manufacturer's protocol. PCR amplification was performed in a volume of $50 \mu \mathrm{l}$ containing $200 \mathrm{ng}$ cDNA, $25 \mathrm{pmol} / \mathrm{l}$ of each primer, $0.25 \mathrm{mmol} / 1$ deoxyribonucleotide triphosphates, $5 \mu 110 \mathrm{X}$ buffer, $21.5 \mathrm{mmol} / \mathrm{l} \mathrm{MgCl}$ and 2.0 U Taq HS polymerase (Takara Biotechnology Co., Ltd.). Primers for the PCR were as follows: TNF- $\alpha$ forward, 5'-CAAACCACCAAGCAGAGGAGC-3' and reverse, 5'-CAA AGTGAGCTTGCCCGGACT-3'; TACE forward, 5'-CAC TTT GGTGCCTTTCGTCC-3' and reverse, 5'-AGCTCGCCT CTT CGCTCGAC-3'; and $\beta$-actin forward, 5'-ATCCGTAAA GACCTCTATGC-3' and reverse, 5'-AACGCAGCTCAGTAA CAGTC-3' (BIO Asia Biotechnology Co., Ltd., Shanghai, China). PCR cycling conditions were as follows: An initial denaturation step at $94^{\circ} \mathrm{C}$ for $3 \mathrm{~min}$, followed by 35 cycles of $94^{\circ} \mathrm{C}$ for $30 \mathrm{sec}, 52 / 48^{\circ} \mathrm{C}$ for $30 \mathrm{sec}$ (TNF- $\alpha / \mathrm{TACE}$ ), and $72^{\circ} \mathrm{C}$ for $60 \mathrm{sec}$. The PCR products were subjected to agarose gel electrophoresis and the abundance of each mRNA was normalized against $\beta$-actin using the Gel Doc 1000 UV Gel Imaging system (Bio-Rad Laboratories, Inc., Hercules, CA, USA).

Statistical analysis. SPSS software, version 10.0 (SPSS, Inc., Chicago, IL, USA) was used to conduct statistical analyses. Data are presented as the mean \pm standard deviation. Homogeneity of variance was evaluated using the Levene's test. In the case of homogeneity, one-way analysis of variance was conducted, followed by pair-wise between- and within-group comparisons using least significant difference (LSD) tests. In the case of heterogeneity, the Games-Howell test was used for analysis. 

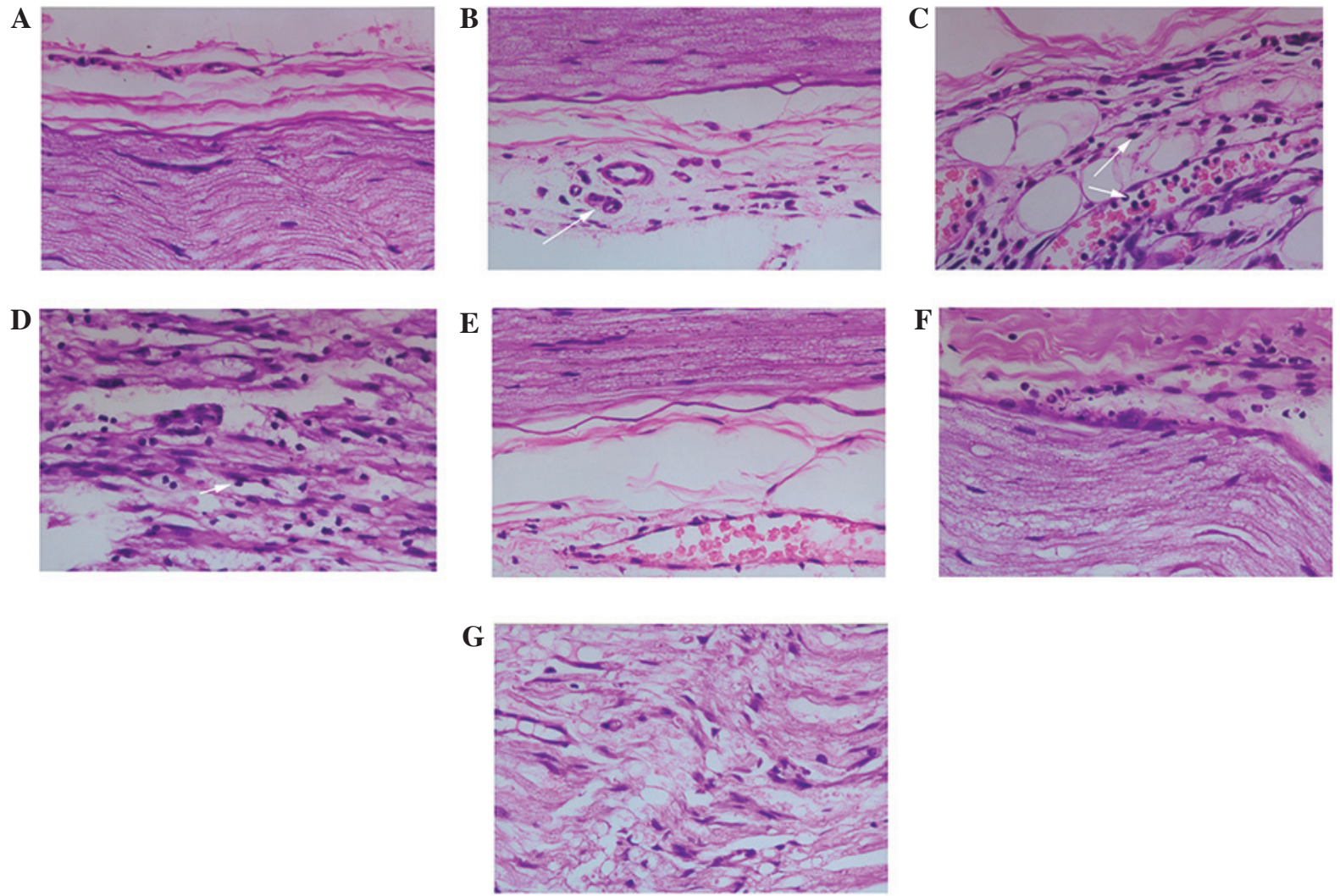

Figure 1. Effects of MK-801 on histological alterations in the rat sciatic nerve (SN) following ischemia/reperfusion (I/R) injury. Histological changes were determined using hematoxylin \& eosin (H\&E) staining (magnification, x400). (A) SN fibers and the outer membrane of blood vessels from a sham-operated rat. (B) SN fibers and the outer membrane of blood vessels from a rat in the I/R group at $6 \mathrm{~h}$ post-reperfusion. Obvious edema around the epineurium, leakage and infiltration of neutrophils around blood vessels, and swelling of nerve fibers (arrow) were observed. (C) SN fibers and the outer membrane of blood vessels from a rat in the I/R group at $24 \mathrm{~h}$ post-reperfusion. Infiltration of neutrophils into the nerve fiber bundle peaks, more severe fiber swelling, and partial demyelination (white arrows) was observed. (D) SN fibers from a rat in the I/R group at 7 days post-reperfusion. Large numbers of infiltrating macrophages and monocytes were observed around the Schwann cells, which was accompanied by extensive axonal demyelination. (E) SN fibers and blood vessels surrounding the epineurium from a rat in the $\mathrm{I} / \mathrm{R}+\mathrm{MK}-801$ group at $6 \mathrm{~h}$ post-reperfusion. Pretreatment with MK- 801 resulted in milder edema around the epineurium, fewer infiltrating neutrophils around blood vessels, and only minor swelling of nerve fibers. (F) SN fibers and blood vessels around the epineurium from a rat in the $\mathrm{I} / \mathrm{R}+\mathrm{MK}-801$ group at $24 \mathrm{~h}$ post-reperfusion. As compared with the $\mathrm{I} / \mathrm{R}$ group rats at the same post-reperfusion time point, there was less extensive axonal swelling and edema of the endoneurium and myelin sheath, fewer infiltrating neutrophils, and no observable demyelination. (G) SN from a rat in the $\mathrm{I} / \mathrm{R}+\mathrm{MK}-801$ group at 7 days post-reperfusion. As compared with the I/R group rats at the same post-reperfusion time point, swelling of myelin sheathes, infiltration of macrophages and monocytes around Schwann cells, and demyelination were less severe.

$\mathrm{P}<0.05$ was considered to indicate a statistically significant difference.

\section{Results}

Effects of MK-801 pretreatment on I/R-induced iNOS activity in the $S N$. The SNs isolated from the sham-operated group exhibited low levels of iNOS activity (Table I). In the I/R group, the activity of iNOS was not significantly different to the sham group immediately following reperfusion $(0 \mathrm{~h}$ time point; $\mathrm{P}>0.05$ ); however, it gradually increased thereafter $(\mathrm{P}<0.05$ at $\geq 6 \mathrm{~h})$, and peaked at $24 \mathrm{~h}$ following reperfusion $(\mathrm{P}<0.01$; Table I). The activity of iNOS was decreased in the I/R group at $>24 \mathrm{~h}$ post-reperfusion; however, it remained significantly different to the sham group at 7 days post-reperfusion $(\mathrm{P}<0.05$; Table I). In the $\mathrm{I} / \mathrm{R}+\mathrm{MK}-801$ group, the activity of iNOS followed the same temporal course as the I/R group; however, the activity was markedly lower at each time point, and the differences were significant at 12,24 , $72 \mathrm{~h}$, and 7 days post-reperfusion, as compared with the I/R group (all $\mathrm{P}<0.05$ ).
Effects of MK-801 pretreatment on I/R-induced increases in plasma NO and MDA levels. As compared with the sham group, the I/R group exhibited elevated plasma levels of NO immediately following reperfusion, which peaked at $24 \mathrm{~h}$ post-reperfusion, and remained significantly elevated at 7 days post-reperfusion $(\mathrm{P}<0.05$; Table I). The mean plasma levels of $\mathrm{NO}$ in the I/R+MK-801 group followed the same general trend; however, the plasma levels of NO were always lower in the I/R+MK-801 group, as compared with the I/R group, and were significantly lower at $6,12,24,72 \mathrm{~h}$, and 7 days post-reperfusion $(\mathrm{P}<0.05$; Table I).

Plasma concentrations of MDA were significantly elevated in the $\mathrm{I} / \mathrm{R}$ group at all time points, as compared with the sham group $(\mathrm{P}<0.05$; Table $\mathrm{I})$. In addition, the $\mathrm{I} / \mathrm{R}+\mathrm{MK}-801$ group exhibited lower mean plasma MDA levels at all time points, as compared with the I/R group, and were significantly different at $6,12,24$ and $72 \mathrm{~h}$ post-reperfusion (all $\mathrm{P}<0.05$; Table I).

A temporal correlation between iNOS activity and levels of NO suggested that iNOS activity is the primary source of plasma NO, and lower plasma levels of MDA and NO in the 

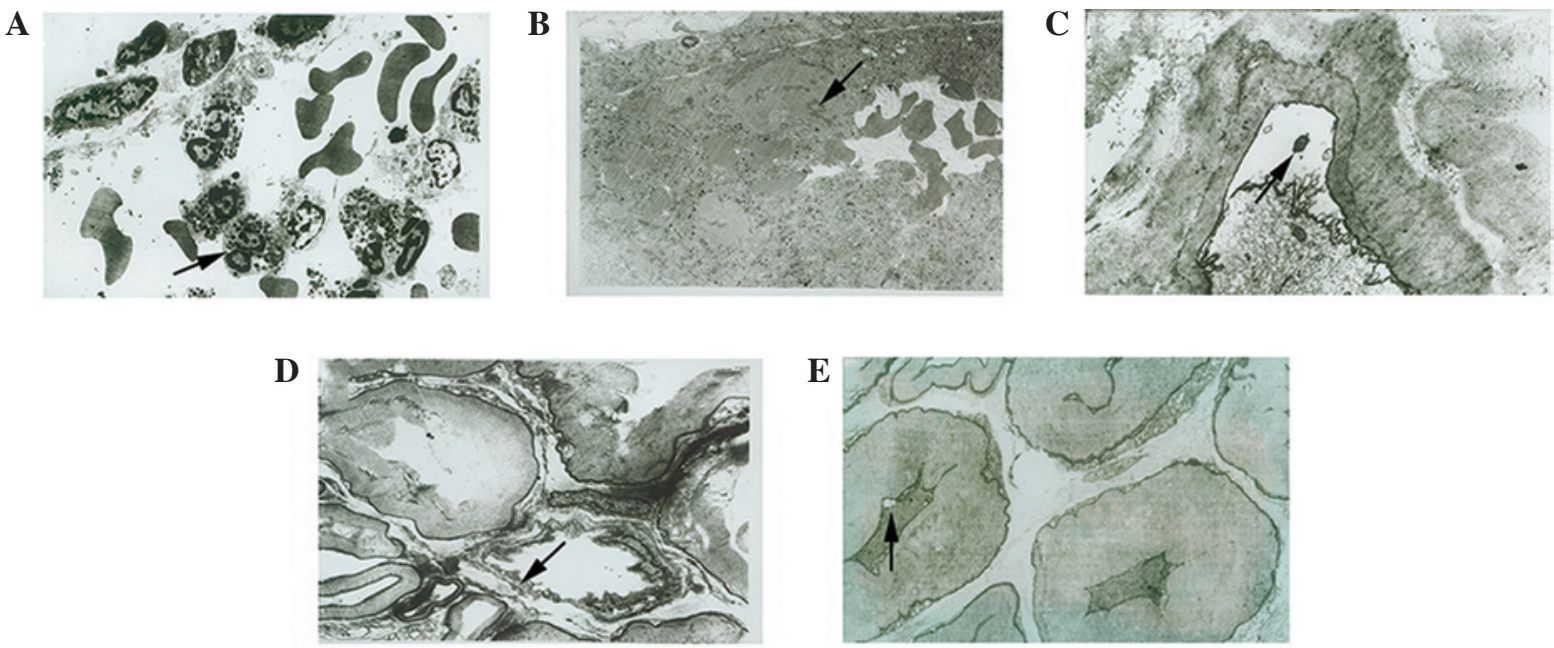

Figure 2. Effects of MK-801 on ultrastructural changes in the rat sciatic nerve (SN) following ischemia/reperfusion (I/R) injury. The ultramicrostructure was examined using transmission electron microscopy. (A) Blood vessels surrounding the epineurium from a rat in the I/R group at $24 \mathrm{~h}$ post-reperfusion (magnification, $\mathrm{x} 7,000$ ). Neutrophils (black arrow) were adhered to the blood vessel wall and were infiltrating the nerve. (B) Blood vessels surrounding the epineurium from a rat in the I/R group at $24 \mathrm{~h}$ post-reperfusion (magnification, x7,000). Thrombosis (black arrow), platelet aggregation and deformed erythrocytes were detected. (C) Myelin sheathes in a SN from a rat in the I/R group at $24 \mathrm{~h}$ post-reperfusion (magnification, x10,000). Fissures (black arrow) were detected between the axonal membrane and myelin sheath. (D) Myelin sheathes in a SN from a rat in the I/R group at $24 \mathrm{~h}$ post-reperfusion (magnification, $\mathrm{x} 7,000$ ). Erosion (black arrow) was detected. (E) Myelin sheathes in the SN from a rat in the I/R + MK-801 group at 24 h post-reperfusion (magnification, $\mathrm{x} 8,000$ ). Myelin sheathes exhibited mild shrinkage and occasional small fissures (black arrow) were observed between the axonal membrane and myelin sheath.

I/R+MK-801 group, as compared with the I/R group, indicated that MK-801 may reduce MDA levels by reducing the concentration of plasma NO.

Effects of MK-801 on I/R-induced histological changes in the $S N$. SNs from the sham group exhibited no significant histological alterations after $5 \mathrm{~h}$ of ischemia, with only moderate swelling of endothelial cells and edema around the blood vessels (Fig. 1A). At $6 \mathrm{~h}$ post-reperfusion, neutrophils were adhered to the blood vessel walls and had infiltrated the surrounding endoneurium and epineurium (Fig. 1B). At $12 \mathrm{~h}$ post-reperfusion, further neutrophil adherence and infiltration was observed. In addition, the myelin sheathes were vesicular in appearance, and degranulation of neutrophils was detected. At $24 \mathrm{~h}$ post-reperfusion, the leakage of neutrophils out of blood vessels and infiltration into the SN reached a peak, and this was accompanied by detection of neutrophils around myelinated fibers, adherence of monocytes to vessels surrounding the endoneurium, and obvious edema and swelling of the endoneurium and myelin sheathes (Fig. 1C). At 72 h post-reperfusion, marked infiltration of monocytes was observed, macrophages were detected around Schwann cells and axon demyelination was observed. At 7 days post-reperfusion, numerous infiltrated macrophages and monocytes were detected, and this was accompanied by widespread demyelination and edema of the myelin sheathes, endoneurium and endothelial cells (Fig. 1D).

No significant morphological alterations were observed in the SNs from rats in the I/R+MK-801 group immediately following reperfusion, and there was less edema, as compared with the I/R group. At 6 and $12 \mathrm{~h}$ post-reperfusion, fewer adherent and infiltrated neutrophils were observed, as compared with the I/R group (Fig. 1E). At $24 \mathrm{~h}$ post-reperfusion in the I/R+MK-801 group only mild inflammation was observed, with comparatively less edema and swelling of the endoneurium and myelin sheathes and no detectable demyelination (Fig. 1F). At $72 \mathrm{~h}$ and 7 days post-reperfusion, there remained fewer infiltrated monocytes surrounding Schwann cells and less demyelination, as compared with the I/R group (Fig. 1G).

Effects of MK-801 on I/R-induced ultrastructural changes in the $S N$. SNs isolated from the sham, I/R and I/R+MK-801 groups were also examined by TEM. Consistent with the histopathological observations, inflammatory cells were detected in the blood vessels and surrounding the epineurium of the SNs from the I/R group, which was accompanied by the adherence of neutrophils to the blood vessel walls and the leakage of neutrophils out of blood vessels. In addition, spaces between endothelial cells were broadened, which indicated a loss of blood vessel integrity (Fig. 2A). Certain neutrophils were observed to be distant from the blood vessel walls, and small numbers of lymphocytes were shown to surround detached collagen fibers. Furthermore, deformed erythrocytes were observed within various blood vessels, and signs of thrombosis and platelet aggregation, with concomitant narrowing or even complete blockage of blood vessels by fibrin and blood cells, was detected (Fig. 2B). Small numbers of infiltrating neutrophils and other inflammatory cells were detected around nerve bundles, and fissures of various sizes due to detached myelin were observed. In addition, swelling of various axons was observed and irregularly shaped effusion cavities were shown to have formed due to the separation of myelin sheathes from axons (Fig. 2C). Certain myelin sheathes showed signs of erosion, and various axons exhibited swollen mitochondria and endoplasmic reticulum (ER). Swollen mitochondria were also observed in the Schwann cells (Fig. 2D).

$\mathrm{I} / \mathrm{R}$ injury-induced ultrastructural changes appeared to be less severe in the SNs from the I/R+MK-801 group rats, 

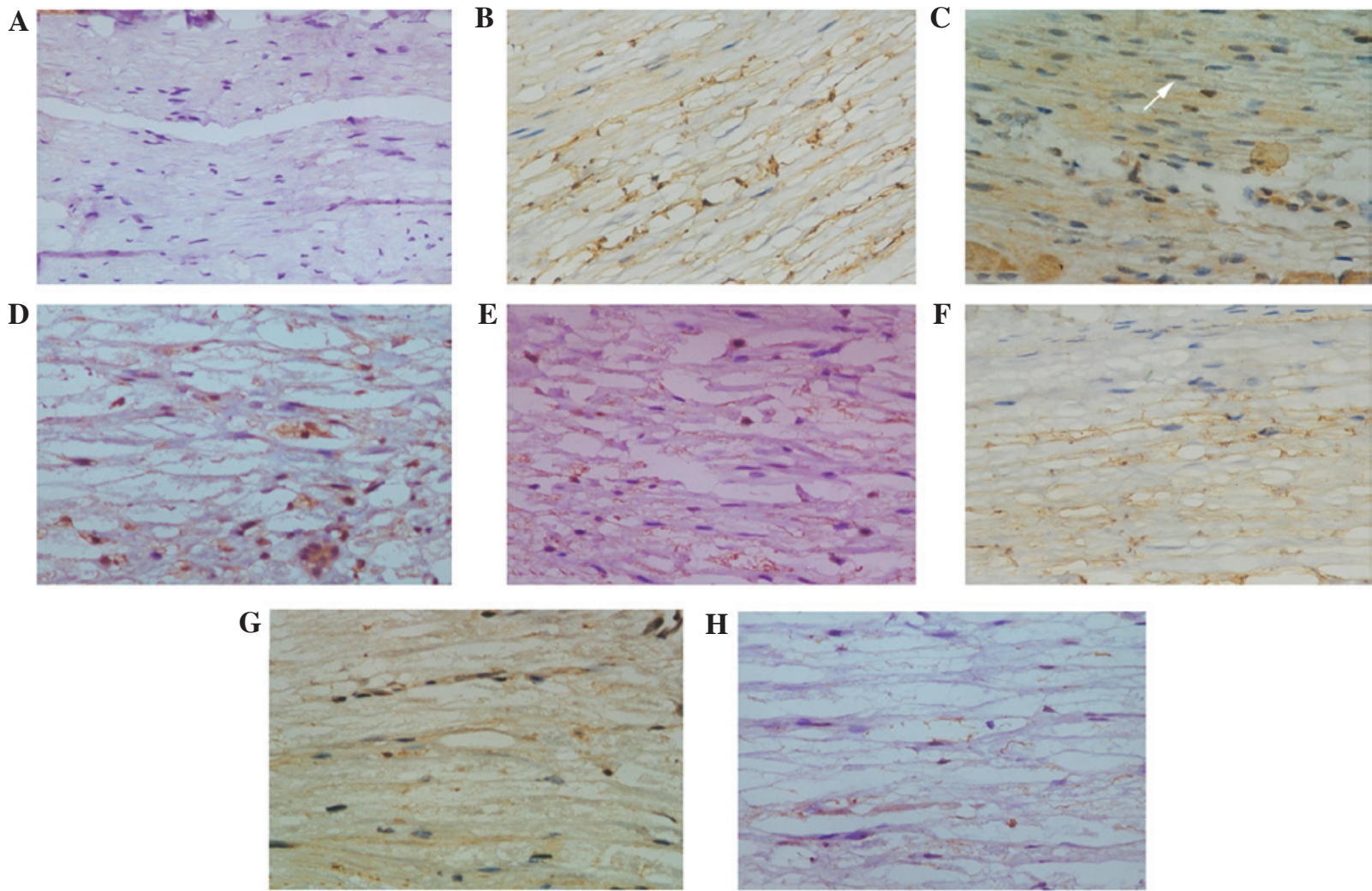

Figure 3. Effects of MK-801 on tumor necrosis factor (TNF)- $\alpha$ protein expression levels in the rat sciatic nerve (SN) following ischemia/reperfusion (I/R) injury. Protein expression levels of TNF- $\alpha$ were determined using immunohistochemistry (magnification, $\mathrm{x} 400$ ). (A) No TNF- $\alpha$ expression was detected in the Schwann cells derived from the SN of a sham-operated rat. (B) Moderate protein expression levels of TNF- $\alpha$ were detected in the Schwann cells derived from the SN fiber of a rat in the $12 \mathrm{~h}$ post-reperfusion I/R subgroup. (C) Higher protein expression levels of TNF- $\alpha$ were detected in the Schwann cells derived from the SN of a rat in the $24 \mathrm{~h}$ post-reperfusion I/R subgroup. (D) Numerous inflammatory cells had infiltrated the area surrounding the Schwann cells and moderate protein expression levels of TNF- $\alpha$ were detected in the SN of a rat in the $72 \mathrm{~h}$ post-reperfusion I/R subgroup. (E) Widespread demyelination and mild-to-moderate TNF- $\alpha$ protein expression levels in Schwann cells were detected in the SN derived from a rat in the 7 days post-reperfusion I/R subgroup. (F) A SN from a rat in the I/R + MK-801 group at $12 \mathrm{~h}$ post-reperfusion exhibited mild-to-moderate TNF- $\alpha$ protein expression levels in Schwann cells. (G) A SN from a rat in the I/R + MK-801 group at $24 \mathrm{~h}$ post-reperfusion exhibited markedly fewer infiltrating cells, as compared with the SN derived from $\mathrm{I} / \mathrm{R}$ rats at the same time point post-reperfusion. Moderate protein expression levels of TNF- $\alpha$ expression were observed. (H) A SN derived from a rat in the $\mathrm{I} / \mathrm{R}+\mathrm{MK}-801$ group at 7 days post-reperfusion. As compared with the SNs derived from the I/R rats, the extent of demyelination was markedly reduced and Schwann cells exhibited only low protein expression levels of TNF- $\alpha$.

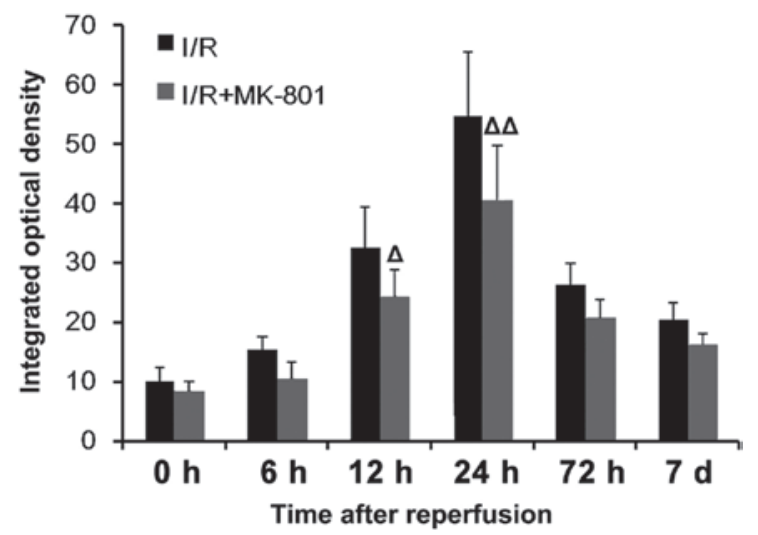

Figure 4. Protein expression levels of tumor necrosis factor- $\alpha$ in the various treatment subgroups were quantified using the integrated optical density method, and are presented as the mean \pm standard deviation $(\mathrm{n}=6) .{ }^{\Delta} \mathrm{P}<0.05$, ${ }^{\Delta \Delta} \mathrm{P}<0.01$ vs. the $\mathrm{I} / \mathrm{R}$ group. $\mathrm{I} / \mathrm{R}$, ischemia reperfusion.

which exhibited fewer infiltrating inflammatory cells, swollen mitochondria in axons and Schwann cells, and less severe ER swelling. In addition, no thrombosis, platelet aggregation, or clots were observed in the blood vessels of the SNs from the I/R+MK-801 group. Furthermore, there were no signs of demyelination, including fissures and effusion cavities, between axons and myelin sheathes (Fig. 2E).

Effects of MK-801 on I/R-induced TNF- $\alpha$ protein expression in the $S N$. SNs isolated from the sham group exhibited no detectable expression of TNF- $\alpha$ in the Schwann cells (Fig. 3A). At the initiation of reperfusion following 5 -h ischemia, low protein expression levels of TNF- $\alpha$ were detected in the Schwann cells. Concomitant with inflammatory cell accumulation at the vessel walls and infiltration at 6-12 h post-reperfusion, the protein expression levels of TNF- $\alpha$ increased rapidly (Fig. 3B), and peaked at $24 \mathrm{~h}$ post-reperfusion (Fig. 3C). At $72 \mathrm{~h}$ post-reperfusion, the migration of neutrophils had ceased, macrophages had infiltrated and were surrounding the Schwann cells, demyelination was detected, and the protein expression levels of TNF- $\alpha$ were gradually returned to baseline levels (Fig. 3D). At 7 days post-reperfusion, widespread general demyelination was detected and the protein expression levels of TNF- $\alpha$ had remained 2-fold higher, as compared with baseline levels (Fig. 3E). 

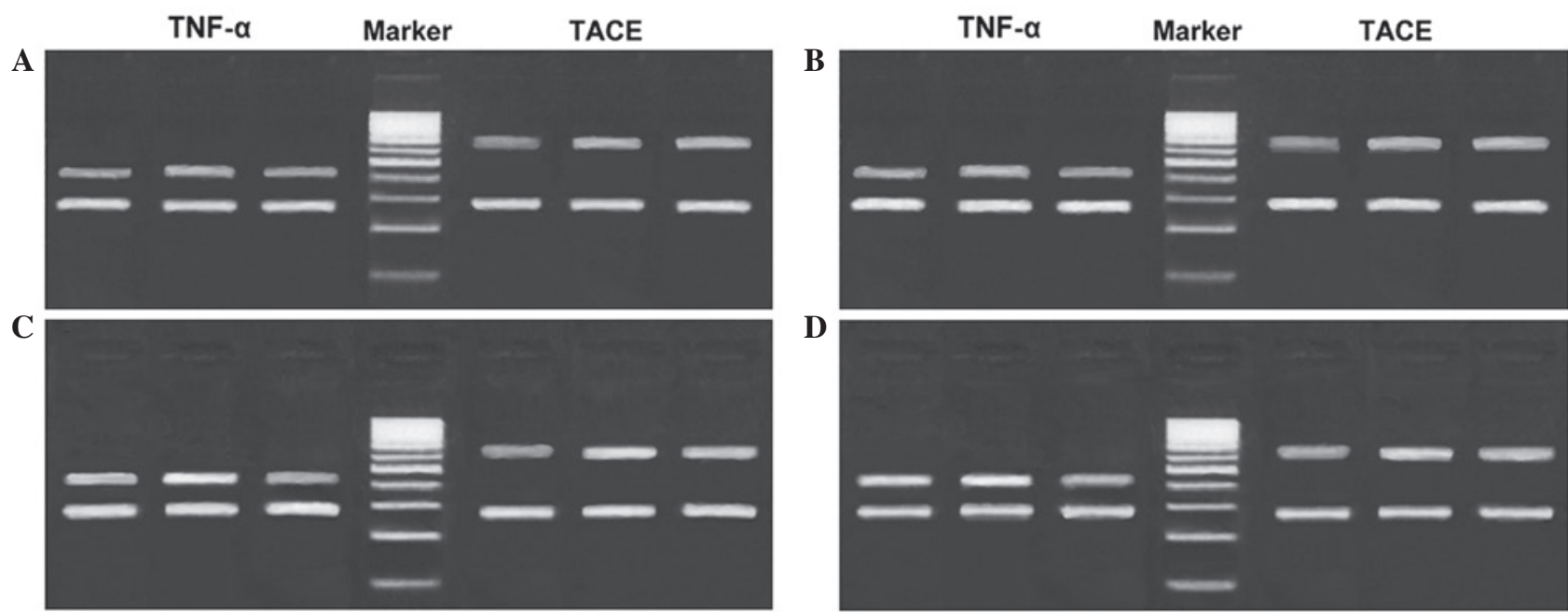

$\mathbf{E}$

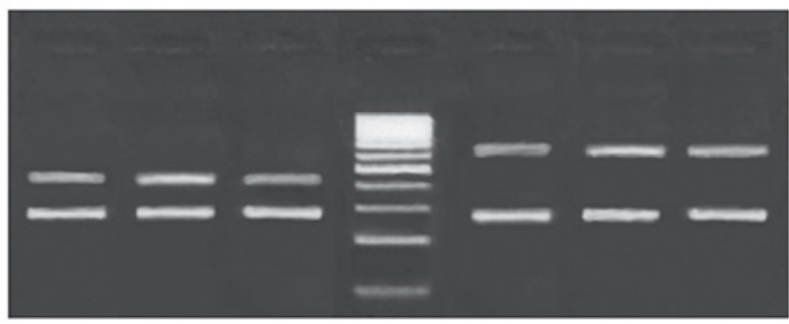

I/R+MK-801 I/R sham

sham I/R I/R+MK-801 I/R+MK-801 I/R sham
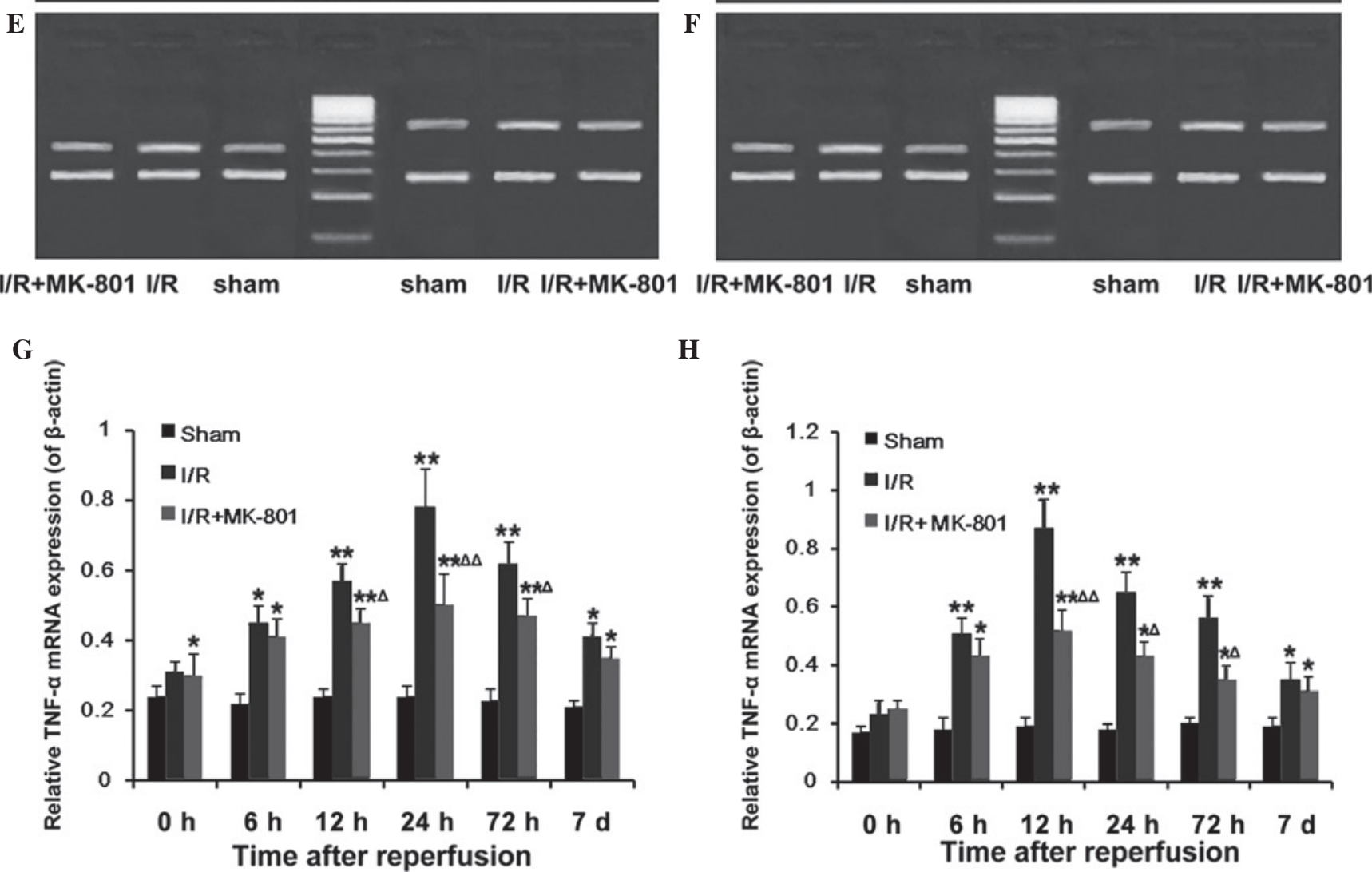

$\mathbf{H}$

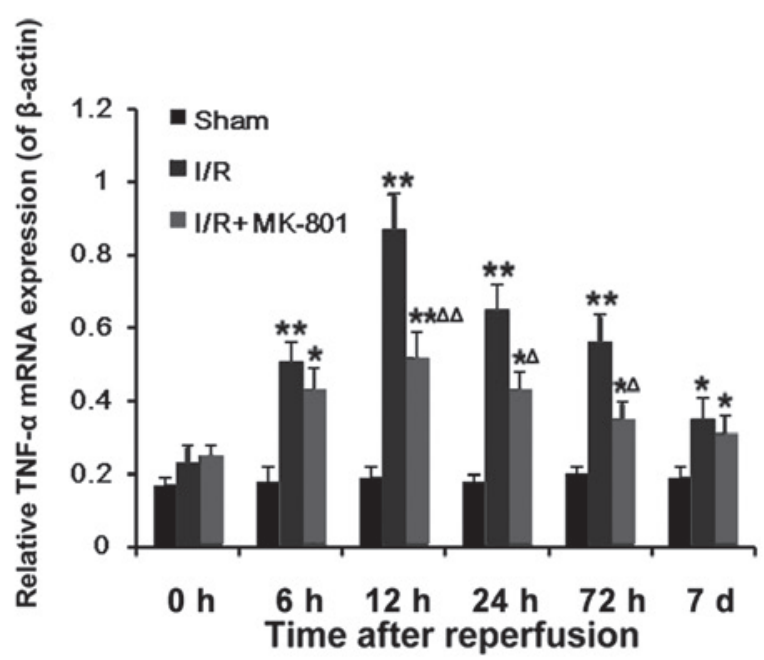

Figure 5. Effects of MK-801 on TNF- $\alpha$ and TACE mRNA expression levels in the rat sciatic nerve (SN) following ischemia/reperfusion (I/R) injury. TNF- $\alpha$ and TACE mRNA expression levels were determined using reverse transcription-quantitative polymerase chain reaction and are expressed relative to $\beta$-actin. Agarose gel images showing TNF- $\alpha$ and TACE mRNA expression levels in the SN homogenates at (A) 0, (B) 6, (C) 12 , (D) 24 and (E) $72 \mathrm{~h}$ and (F) 7 days post-reperfusion. $\beta$-actin $=280 \mathrm{bp}$; TNF- $\alpha=402 \mathrm{bp}$; TACE $=624 \mathrm{bp}$. Relative (G) TNF- $\alpha$ and (H) TACE mRNA expression levels are presented as the mean \pm standard deviation $(\mathrm{n}=6) .{ }^{*} \mathrm{P}<0.05,{ }^{* *} \mathrm{P}<0.01$ vs. the sham-operated group; ${ }^{\Delta \mathrm{P}}<0.05,{ }^{\Delta \Delta} \mathrm{P}<0.01$ vs. the $\mathrm{I} / \mathrm{R}$ group. TNF- $\alpha$, tumor necrosis factor- $\alpha$; TACE, TNF- $\alpha$-converting enzyme.

MK-801 pre-treatment markedly reduced the protein expression levels of TNF- $\alpha$ during reperfusion (Fig. 3F-H), as demonstrated by a reduction in the number of cells positive for TNF- $\alpha$ and the intensity of staining. IOD measurements demonstrated that the protein expression levels TNF- $\alpha$ were significantly reduced at 12 and $24 \mathrm{~h}$ post-reperfusion, as compared with the I/R group (Fig. 4).

Effect of MK-801 on I/R-induced mRNA expression of TNF- $\alpha$ and TACE in the $S N$. Following $5 \mathrm{~h}$ of ischemia, the mRNA expression levels of TNF- $\alpha$ in the SNs from the I/R group rats were not significantly different, as compared with the sham group $(\mathrm{P}>0.05)$. However, reperfusion significantly increased the mRNA expression levels of TNF- $\alpha$, which peaked at $24 \mathrm{~h}$ post-reperfusion, prior to decreasing to a level $\sim 2$-fold above the baseline at 7 days post-reperfusion (Fig. 5A-G). Similarly, the mRNA expression levels of TACE were increased during reperfusion, and peaked at $12 \mathrm{~h}$ post-reperfusion, prior to returning to the baseline at 7 days post-reperfusion (Fig. 5A-F and H). Pretreatment with MK-801 markedly 
reduced the mRNA expression levels of TNF- $\alpha$ and TACE throughout the reperfusion period, with significant differences at $12-72 \mathrm{~h}$ post-reperfusion $(\mathrm{P}<0.05)$.

\section{Discussion}

Glutamate neurotoxicity (excitotoxicity) is a seminal upstream event in the pathogenesis of $\mathrm{I} / \mathrm{R}$-induced neuronal injury in the CNS (6). NMDAR is a major contributor to this process, since it is the predominant calcium-permeable glutamate receptor isoform and thus the major link between the accumulation of extracellular glutamate from dysregulated synaptic release and reverse transport (34-37), and intracellular neurodegenerative pathways triggered by intracellular calcium elevation, including proteolytic and free radical damage $(5,6,38)$. The results of the present study suggested that NMDAR stimulation may be a critical early trigger of I/R-induced neuronal injury in the PNS. Notably, MK-801 pre-treatment was able to inhibit numerous neurodegenerative downstream pathways in the SN, including edema, cell swelling, mitochondrial damage, immune cell infiltration and demyelination. Activation of TNF- $\alpha$, TACE, and iNOS, which are mediators of nerve inflammation and oxidative stress, may be a key NMDAR-dependent intermediate step in this process, as rescue from I/R-induced injury was associated with reduced iNOS, TNF- $\alpha$, and TACE expression levels, as well as decreased accumulation of MDA, which is an indicator of oxidative stress.

The tissue microenvironment surrounding peripheral nerves is distinct from the white matter of the CNS; thus major differences in the underlying pathogenesis of I/R-induced nerve injury in the CNS and the PNS may be expected. For example, the source of the excitotoxic glutamate (synaptic vs. non-synaptic), the role of the supporting cells (astrocytes vs. Schwann cells), the immunoresponse, and the neuroprotective and cytotoxic intermediates are distinct (39). However, the rat $\mathrm{SN}$ exhibited a similar sequence of pathogenic processes following I/R, as compared with the CNS, including early edema and cell swelling, followed by delayed inflammation, axonal degeneration and demyelination. Notably, these processes were blocked or reduced in severity by MK-801 pre-treatment; thus suggesting that early NMDAR activation may be involved in the events leading to I/R injury $(40,41)$.

In a previous study, peripheral application of MK-801 was shown to prevent peripheral nerve damage (30), whereas activation of peripheral glutamate receptors was shown to induce peripheral nerve damage or dysfunction (30). For example, the thermogenic flare induced by subdermal injection of the bee venom toxin was associated with local extracellular glutamate accumulation and was attenuated by co-injection with MK-801 (12); thus suggesting a role for local NMDARs on sensory afferents as opposed to spinal NMDARs. Similarly, peripheral glutamate released by damaged tissues and reverse transport or glutamate influx from damaged capillaries, may have induced SN damage during and following ischemia (37). Sodium influx upon NMDAR activation may lead to axonal swelling by osmotic water movement, consistent with the observed relief by MK-801 (42). Calcium influx associated with NMDAR activation and ischemic-depolarization may initiate a chain of biochemical events leading to axonal damage, including calcium-dependent calcium release (43). At least within central axons, a form of excitation-calcium coupling analogous to that in muscle cells may exist, which induces calcium release from ryanodine-sensitive stores $(5,44)$. In addition, the ER is a major calcium store (45), and the ultrastructural investigations conducted in the present study detected signs of axonal ER damage, possibly resulting in calcium egress. In turn, dysregulated calcium may activate proteases that damage the axonal cytoskeleton, resulting in disruption of axonal transport with concomitant inhibition of retrograde growth factor signaling (46). These same processes may also be triggered at the proximal end of the nerve (closer to the spinal cord), since glutamate within the dorsal root ganglia was enhanced by SN injury, and cells within the dorsal root ganglia responded to direct application of various glutamate receptor agonists, including NMDA (13).

NO may relieve or exacerbate ischemic neural injury, depending on context (47). As a vasorelaxant, NO synthesized by neuronal NOS and iNOS may rescue tissue by promoting reperfusion (48). Notably, ischemic preconditioning involves iNOS induction (49). Conversely, in previous studies, silencing iNOS exerted neuroprotective effects against neurodegenerative diseases by reducing microglial activation (50) and/or oxidative stress (51). The present study demonstrated a temporal correlation between iNOS activation and lipid peroxidation during reperfusion; thus suggesting that iNOS was a major source of free radicals in the reperfused $\mathrm{SN}$ axons.

Comparable to NO, TNF- $\alpha$ may exert protective and deleterious effects during ischemia $(52,53)$, with low levels mediating preconditioning, possibly by enhancing the sensitivity of neurons to growth factors. However, TNF- $\alpha$ is a central inflammatory mediator and higher concentrations have previously been shown to be deleterious (26). In the present study, the reduction of TNF- $\alpha$ levels by blocking NMDA receptors and reducing the expression levels of TACE markedly suppressed the neuroinflammatory response, leading to maintenance of the integrity of blood vessels, axonal myelination, mitochondria, ER and Schwann cells during reperfusion.

The present study included a number of limitations. First, the clamping of the femoral artery induced $I / R$ in the entire limb; although only the SN was sampled and examined, the effects of I/R on the entire limb may have affected the present results. Second, the H\&E sections and electron microscopy examinations were analyzed subjectively. Third, the experimental approach did not allow the determination of exact cause-to-effect relationships, nor clarify the specific mechanisms underlying peripheral nerve I/R injury. Furthermore, the lack of homology between rodent and human anatomy, physiology and response to injury and inflammation may limit the relevance of the results of the present study to humans. Further studies are required in order to address these issues and to improve the current understanding of peripheral nerve I/R injury.

In conclusion, the present study demonstrated that systemic injection of the NMDA receptor antagonist MK-801 was able to protect the rat $\mathrm{SN}$ against I/R injury, including attenuating immune cell infiltration and demyelination, possibly by inhibiting the activation of TNF- $\alpha$ and reducing the expression levels of iNOS in the SN. 


\section{Acknowledgements}

The authors would like to thank the Fujian Provincial Hospital Emergency Center of Fujian Province and the Provincial Clinical Medical College of Fujian Medical University for their assistance. The present study was supported by the National Key Clinical Specialist Construction Programs of China (grant no. 2006-1-45).

\section{References}

1. Hajek V, Dussart C, Klack F, Lamy A, Martinez JY, Lainé P, Mazurier L, Guilloton L and Drouet A: Neuropathic complications after 157 procedures of continuous popliteal nerve block for hallux valgus surgery. A retrospective study. Orthop Traumatol Surg Res 98: 327-333, 2012.

2. Nassr A, Eck JC, Ponnappan RK, Zanoun RR, Donaldson WF III and Kang JD: The incidence of C5 palsy after multilevel cervical decompression procedures: A review of 750 consecutive cases. Spine (Phila Pa 1976) 37: 174-178, 2012.

3. Pavlovic D, Kocic G, Cvetkovic T, Simic D, Basic J and Zivanovic D: Biomarkers of oxidative stress and endothelial dysfunction after tourniquet release in children. Physiol Res 60 (Suppl 1): S137-S145, 2011.

4. Marin PC, Im MJ, Girotto JA, Borschel G and Bickel KD: Effects of hydroxyethyl-starch-bound deferoxamine on ischemia/reperfusion injury in chronic nerve compression. J Reconstr Microsurg 14: 485-490, 1998.

5. Stirling DP and Stys PK: Mechanisms of axonal injury: Internodal nanocomplexes and calcium deregulation. Trends Mol Med 16: 160-170, 2010.

6. Puyal J, Ginet V and Clarke PG: Multiple interacting cell death mechanisms in the mediation of excitotoxicity and ischemic brain damage: A challenge for neuroprotection. Prog Neurobiol 105: $24-48,2013$.

7. Zhou S, Bonasera L and Carlton SM: Peripheral administration of NMDA, AMPA or KA results in pain behaviors in rats Neuroreport 7: 895-900, 1996.

8. Ushida T, Tani T, Kawasaki M, Iwatsu $\mathrm{O}$ and Yamamoto $\mathrm{H}$ Peripheral administration of an N-methyl-D-aspartate receptor antagonist (MK-801) changes dorsal horn neuronal responses in rats. Neurosci Lett 260: 89-92, 1999.

9. Ro JY: Contribution of peripheral NMDA receptors in craniofacial muscle nociception and edema formation. Brain Res 979: $78-84,2003$

10. Jang JH, Kim DW, Sang Nam T, Se Paik K and Leem JW: Peripheral glutamate receptors contribute to mechanical hyperalgesia in a neuropathic pain model of the rat. Neuroscience 128: 169-176, 2004

11. Lam DK, Sessle BJ, Cairns BE and Hu JW: Peripheral NMDA receptor modulation of jaw muscle electromyographic activity induced by capsaicin injection into the temporomandibular joint of rats. Brain Res 1046: 68-76, 2005.

12. Iwashita N, Nosaka S and Koyama N: Involvement of peripheral NMDA receptor in melittin-induced thermographic flare. Neurochem Res 37: 2222-2228, 2012.

13. Kung LH, Gong K, Adedoyin M, Ng J, Bhargava A, Ohara PT and Jasmin L: Evidence for glutamate as a neuroglial transmitter within sensory ganglia. PLoS One 8: e68312, 2013.

14. He K, Nukada H, McMorran PD and Murphy MP: Protein carbonyl formation and tyrosine nitration as markers of oxidative damage during ischaemia-reperfusion injury to rat sciatic nerve. Neuroscience 94: 909-916, 1999.

15. Nagamatsu M, Schmelzer JD, Zollman PJ, Smithson IL, Nickander KK and Low PA: Ischemic reperfusion causes lipid peroxidation and fiber degeneration. Muscle Nerve 19: 37-47, 1996.

16. McCord JM: Oxygen-derived free radicals in postischemic tissue injury. N Engl J Med 312: 159-163, 1985.

17. Schmelzer JD, Zochodne DW and Low PA: Ischemic and reperfusion injury of rat peripheral nerve. Proc Natl Acad Sci USA 86: $1639-1642,1989$.

18. Iida H, Nagasaka T, Shindo K and Shiozawa Z: Effect of the free radical scavenger edaravone on peripheral nerve ischemia-reperfusion injury. Muscle Nerve 40: 582-588, 2009.

19. Lefebvre RA: Nitric oxide in the peripheral nervous system. Ann Med 27: 379-388, 1995.
20. Cárdenas A, Moro MA, Hurtado O, Leza JC, Lorenzo P, Castrillo A, Bodelón OG, Boscá L and Lizasoain I: Implication of glutamate in the expression of inducible nitric oxide synthase after oxygen and glucose deprivation in rat forebrain slices. J Neurochem 74: 2041-2048, 2000.

21. Yu WH: Nitric oxide synthase in motor neurons after axotomy J Histochem Cytochem 42: 451-457, 1994.

22. Qi WN, Yan ZQ, Whang PG, Zhou Q, Chen LE, Seaber AV, Stamler JS and Urbaniak JR: Gene and protein expressions of nitric oxide synthases in ischemia-reperfused peripheral nerve of the rat. Am J Physiol Cell Physiol 281: C849-C856, 2001.

23. Chen XM, Chen HS, Xu MJ and Shen JG: Targeting reactive nitrogen species: A promising therapeutic strategy for cerebral ischemia-reperfusion injury. Acta Pharmacol Sin 34: 67-77, 2013.

24. Shin SJ, Qi WN, Cai Y, Rizzo M, Goldner RD, Nunley JA II and Chen LE: Inhibition of inducible nitric oxide synthase promotes recovery of motor function in rats after sciatic nerve ischemia and reperfusion. J Hand Surg Am 30: 826-835, 2005.

25. Zhang RL, Zhang ZG and Chopp M: Targeting nitric oxide in the subacute restorative treatment of ischemic stroke. Expert Opin Investig Drugs 22: 843-851, 2013.

26. Wagner R and Myers RR: Endoneurial injection of TNF-alpha produces neuropathic pain behaviors. Neuroreport 7: 2897-2901, 1996.

27. Niu YL, Guo Z and Zhou RH: Up-regulation of TNF-alpha in neurons of dorsal root ganglia and spinal cord during coronary artery occlusion in rats. Cytokine 47: 23-29, 2009.

28. Stübgen JP: Tumor necrosis factor-alpha antagonists and neuropathy. Muscle Nerve 37: 281-292, 2008.

29. Lovering F and Zhang Y: Therapeutic potential of TACE inhibitors in stroke. Curr Drug Targets CNS Neurol Disord 4: 161-168, 2005.

30. Kleinschnitz C, Brinkhoff J, Zelenka M, Sommer C and Stoll G: The extent of cytokine induction in peripheral nerve lesions depends on the mode of injury and NMDA receptor signaling. J Neuroimmunol 149: 77-83, 2004.

31. Ozyurt E, Graham DI, Woodruff GN and McCulloch J: Protective effect of the glutamate antagonist, MK-801 in focal cerebral ischemia in the cat. J Cereb Blood Flow Metab 8: 138-143, 1988.

32. Corbett D, Evans S, Thomas C, Wang D and Jonas RA: MK-801 reduced cerebral ischemic injury by inducing hypothermia. Brain Res 514: 300-304, 1990.

33. Nouri M, Rahimian R, Fakhfouri G, Rasouli MR, Mohammadi-Rick S, Barzegar-Fallah A, Asadi-Amoli F and Dehpour AR: Ipsilateral common iliac artery plus femoral artery clamping for inducing sciatic nerve ischemia/reperfusion injury in rats: A reliable and simple method. J Brachial Plex Peripher Nerve Inj 3: 27, 2008.

34. Hofmeijer $\mathbf{J}$ and van Putten MJ: Ischemic cerebral damage: An appraisal of synaptic failure. Stroke 43: 607-615, 2012.

35. Chao XD, Fei F and Fei Z: The role of excitatory amino acid transporters in cerebral ischemia. Neurochem Res 35: 1224-1230, 2010.

36. Szydlowska K and Tymianski M: Calcium, ischemia and excitotoxicity. Cell Calcium 47: 122-129, 2010.

37. Grewer C, Gameiro A, Zhang Z, Tao Z, Braams S and Rauen T: Glutamate forward and reverse transport: From molecular mechanism to transporter-mediated release after ischemia. IUBMB Life 60: 609-619, 2008.

38. Ma M: Role of calpains in the injury-induced dysfunction and degeneration of the mammalian axon. Neurobiol Dis 60: 61-79, 2013.

39. Richner M, Ulrichsen M, Elmegaard SL, Dieu R, Pallesen LT and Vaegter CB: Peripheral nerve injury modulates neurotrophin signaling in the peripheral and central nervous system. Mol Neurobiol 50: 945-970, 2014.

40. Mey J and Thanos S: Functional and biochemical analysis of CNS-relevant neurotrophic activity in the lesioned sciatic nerve of adult rats. J Hirnforsch 37: 25-50, 1996.

41. Kim MA and Jeong KY: Chronological changes of mechanical allodynia and spinal microglia activation by an intrathecal injection of MK-801. Neuroreport 24: 585-589, 2013.

42. Yu XM: The Role of Intracellular Sodium in the Regulation of NMDA-Receptor-Mediated Channel Activity and Toxicity. Mol Neurobiol 33: 63-80, 2006

43. Xin WK, Kwan CL, Zhao XH, Xu J, Ellen RP, McCulloch CA and $\mathrm{Yu}$ XM: A functional interaction of sodium and calcium in the regulation of NMDA receptor activity by remote NMDA receptors. J Neurosci 25: 139-148, 2005. 
44. Ouardouz M, Nikolaeva MA, Coderre E, Zamponi GW, McRory JE, Trapp BD, Yin X, Wang W, Woulfe J and Stys PK: Depolarization-induced $\mathrm{Ca} 2+$ release in ischemic spinal cord white matter involves $\mathrm{L}$-type $\mathrm{Ca} 2+$ channel activation of ryanodine receptors. Neuron 40: 53-63, 2003.

45. Sisalli MJ, Secondo A, Esposito A, Valsecchi V, Savoia C, Di Renzo GF, Annunziato L and Scorziello A: Endoplasmic reticulum refilling and mitochondrial calcium extrusion promoted in neurons by NCX1 and NCX3 in ischemic preconditioning are determinant for neuroprotection. Cell Death Differ 21: 1142-1149, 2014.

46. Morfini GA, Burns M, Binder LI, Kanaan NM, LaPointe N, Bosco DA, Brown RH Jr, Brown H, Tiwari A, Hayward L, et al: Axonal transport defects in neurodegenerative diseases. J Neurosci 29: 12776-12786, 2009.

47. Brown GC: Nitric oxide and neuronal death. Nitric Oxide 23: 153-165, 2010.

48. Phillips L, Toledo AH, Lopez-Neblina F, Anaya-Prado R and Toledo-Pereyra LH: Nitric oxide mechanism of protection in ischemia and reperfusion injury. J Invest Surg 22:46-55, 2009.
49. Das M and Das DK: Molecular mechanism of preconditioning. IUBMB Life 60: 199-203, 2008.

50. Li M, Dai FR, Du XP, Yang QD and Chen Y: Neuroprotection by silencing iNOS expression in a 6-OHDA model of Parkinson's disease. J Mol Neurosci 48: 225-233, 2012.

51. Chavez-Valdez R, Martin LJ, Flock DL and Northington FJ: Necrostatin-1 attenuates mitochondrial dysfunction in neurons and astrocytes following neonatal hypoxia-ischemia. Neuroscience 219: 192-203, 2012.

52. Taoufik E, Petit E, Divoux D, Tseveleki V, Mengozzi M, Roberts ML, Valable S, Ghezzi P, Quackenbush J, Brines M, et al: TNF receptor I sensitizes neurons to erythropoietin- and VEGF-mediated neuroprotection after ischemic and excitotoxic injury. Proc Natl Acad Sci USA 105: 6185-6190, 2008.

53. Watters $\mathrm{O}$ and $\mathrm{O}^{\prime}$ Connor JJ: A role for tumor necrosis factor- $\alpha$ in ischemia and ischemic preconditioning. J Neuroinflammation 8: 87, 2011. 